\title{
In-Vitro Assessment of Plankton Production Using Fish Hydrolysate
}

\author{
${ }^{1}$ B.B. Sahu, ${ }^{1}$ B.C. Mohapatra, ${ }^{1}$ N.K. Barik, ${ }^{1}$ H. Sahu, ${ }^{1}$ P. Sahoo, ${ }^{2}$ N.C. Biswal, ${ }^{2}$ P.K. \\ Mohanty, ${ }^{1}$ U.L. Mohanty, ${ }^{1}$ S. K. Mahanta, ${ }^{1}$ P. Jayasankar \\ ${ }^{1}$ ICAR-Central Institute of Freshwater Aquaculture \\ Kausalyaganga, Bhubaneswar - 751002, Odisha, India \\ ${ }^{2}$ Utkal University, Vani vihar, Bhubaneswar-751007, Odisha, India \\ binodbsahu@gmail.com
}

\begin{abstract}
An experiment was conducted in glass jars for four weeks to assess the efficiency of fish hydrolysate $(F H)$ of $0.01,0.05,0.25,1.25$ and $6.25 \mathrm{ml} \mathrm{L} L^{-1}$ concentration on the production and growth of primary producers. $T-3\left(0.25 \mathrm{ml} \mathrm{L} \mathrm{L}^{-1}\right)$ dose of $\mathrm{FH}$ has been found to maintain the water quality parameter to be within optimum level. And it ranged from $\mathrm{pH}$ (6.4-7.7), conductivity (312.0-355.3 $\mu \mathrm{s} / \mathrm{cm})$, alkalinity $(51.3-86.0 \mathrm{mg} / \mathrm{l})$, Hardness (38-86 mg/l), $\mathrm{CO}_{2}(2.0-6.7 \mathrm{mg} / \mathrm{l})$, Ammonia (0-0.3mg/l), Nitrite (0-0.1 mg/l). Around 31 genre of phytoplankton belonging to Chlorophyceae (9), bacillarioyophyceae (8), cyanophyceae (7) and zooplankton (7) were identified. The results show that $\mathrm{FH}$ treatment $0.25 \mathrm{ml} \mathrm{L} \mathrm{L}^{-1}$ in $\mathrm{T}-3$ enhances the phytoplankton and zooplankton production than other jar waters. So it has been recommended for application in aquaculture ponds as a biofertilizer for the well growth of the plankton species.
\end{abstract}

Keywords: Fish hydrolysate, primary productivity, aquaculture pond fertilization, biofertilizer.

\section{INTRODUCTION}

In aquaculture the maintenance of good water quality is of primary importance, so that excellent culture environment, adequate food for optimal fish yield, appraisal of the eutrophic situation and increase in plankton population may be achieved. (Sipauba-Tavares et al, 2006; Sipauba-Tavares 2011).

For Aquaculture pond productivity both organic and inorganic fertilizers are used. Inorganic fertilizers mainly increases the primary production asphytoplankton and organic fertilizers increases the abundance growth of zooplankton, insects larval and other forms of fish food organisms(Jhingran 1983; Olah et al., 1986). Organic wastes contain compounds are capable of promoting plant growth (Day AD and Katterman 1992).Organic fertilizers are often used to promote desirable zooplankton species. Organic fertilizers may be animal manures and oil cakes. Organic fertilizers should have less carbon; nitrogen ratios and have fine particular sizes to allow rapid decomposition (Geiger and Turner, 1990). The organic fertilizers (manures) poultry dropping and cowdung manures are frequently used for the growth of aquatic microphytes (Phyto-zoo plankton) which in turn used as fish food and to regulate water quality characteristics of fish ponds. (Mirza et al., 1990)

Cattle manures and poultry droppings upon addition to fish ponds start decomposition and reduction of dissolved oxygen in fish ponds and responsible for mass mortality of micro-organisms produces unpleasant smell and causes mortality of fish and aquatic organisms (Begum et al., 2012). Basal application of cow dung $10000 \mathrm{~kg} / \mathrm{ha}$ and $1000 \mathrm{~kg} / \mathrm{ha}$ every month in nursery ponds found to be acceptable for better water quality management, fish growth and health parameters.

Phytoplankton is the primary producer, which provides food and oxygen for other organisms. Zooplankton comprising of four major groups, viz., Cladocerans, Copepods, Rotrifers and nauplii are the preferred food materials for the cultured fish species. Zooplankton important to larval fish are classified as rotrifers, cladocerans or copepods. The ability of rotifers and cladocerans to reproduce asexually enables them to react quickly to unfavorable and favorable environmental conditions. (Pennak, 1989)

In larviculture information regarding relative status of plankton communities gives insight into water quality parameters and the possible success or failure of culture system. Best management practices 
are to maintain the high densities of desirable zooplankton species in culture pond until the fish were able to consume commercial feed. (Morris and Mischke, 1999)

There is a direct relationship between nutrient and phytoplankton density and species diversity. It was observed that in low temperature low abundance of phytoplankton and nitrate concentration, although phosphate exhibited an inverse relationship with the growth rate of planktonic organisms. Recent studies have suggested phosphorous as the most limiting nutrient with regard to phytoplankton (Moutin et.al, 2002; Chowduryet.al, 2007).

The plankton community on which the whole aquatic population depends, is largely affected by an interaction of number of limiting factors such as low dissolved oxygen, moderatesulphate, nitrate, phosphate and other factors. (Kumar and Omen, 2009). The nitrogen and phosphorous plays dominant role in controlling the growth and abundance on phytoplankton and freshwater angiosperms. (Hecky and Kilham 1988).

Vermi-compost enriched with biofertilzers as successful organic manure for fish growth and ecofriendly product for aquaculture production (Senthil Kumar et al., 2014). Recently acid hydrolysis of fish wastes has been studied to produce low cost nutrients for the production of lactic acid and low cost protein sources have been produced by ensiling hydrolyzed fish viscera to obtain a suitable medium for lactic acid bacteria. (Gao et.al, 2006)

Fish wastes are also utilized for the fish meal production however this process is costly and heat required for drying makes the fish meal less digestible (Yamamato, 1960). According to Afonoso and Borquez, (2002) fermented broth of fish wastes could be a valuable recourses for agriculture. To date only a few reports on reutilization of biodegraded waste products as liquid fertilizers are available. By using some microorganisms extracted from earthworm viscera, the fish wastes were converted into liquid fertilizers (Kim et al 2010)

Both addition of formic acid to cold offal and fermentation of lactic acid bacteria are effective methods of ensiling this material. Peat extracts with relatively high carbohydrate content were found to be a satisfactory source of carbohydrates for the fermentation of lactic acid bacteria in cod offal silage. However, peat extracts with a relatively low $\mathrm{pH}$ were found to be unsatisfactory as a direct source of acid for ensiling (Martin and Bemiste, 1994). The underutilized fish processing waste cost effectively transformed into fish hydrolysate using a natural fermentation process. Their value has been increased by using them as liquid biofertilizer, feed supplement and bio-organic manures. The values have been reported to be nitrogen- $2.95 \%$, phosphorous $-1.98 \%$, potassium $0.65 \%$, sulphur$1.52 \%$, boron-10.4ppm, calcium-2.24\%, magnesium-1.75\%. (Sahu et al., 2014)

\section{MATERIALS AND METHOD}

\subsection{Study Area}

Research was carried out at ICAR- Central Institute of Freshwater Aquaculture $\left(20^{\circ} 11^{\prime} 10.7\right.$ 'N, $\left.85^{\circ} 51^{\prime} 21.7^{\prime \prime} \mathrm{E}\right)$ at Bhubaneswar, Odisha, India in the month of August to October, 2014. The experiment was conducted in $25 \mathrm{~L}$ glass jars filled with $20 \mathrm{~L}$ filtered pond water filtered with mesh size $125 \mu$. Each glass jar was inoculated with fish hydrolysate (FH) (Sahu et al., 2014) at different concentrations in triplicate (Table 1,2). The culture setup was as follows (A control and five doses in triplicate):

1. Control- Pond water alone

2. $\mathrm{T}_{1^{-}} 0.01 \mathrm{ml} \mathrm{L}^{-1}$

3. $\mathrm{T}_{2^{-}} 0.05 \mathrm{ml} \mathrm{L}^{-1}$

4. $\mathrm{T}_{3}-0.25 \mathrm{ml} \mathrm{L}^{-1}$

5. $\mathrm{T}_{4}-1.25 \mathrm{ml} \mathrm{L}^{-1}$

6. $\mathrm{T}_{5^{-}} 6.25 \mathrm{ml} \mathrm{L}^{-1}$

All the culture jars were aerated till the end of this experiment (28 days). Water quality such as temperature, $\mathrm{pH}$, conductivity, $\mathrm{CO} 2$, Alkalinity, Hardness, Ammonia, Nitrate and Nitrite were measured at weekly intervals by following the standard method. (APHA, 1989) 
In-Vitro Assessment of Plankton Production Using Fish Hydrolysate

Table I. Chemical Composition of fish hydrolysate from processing waste

\begin{tabular}{|l|l|}
\hline Parameter & Composition \\
\hline Total Nitrogen (\%) & $1.51 \pm 0.23$ \\
\hline Total Phosphorous (\%) & $0.52 \pm 0.11$ \\
\hline Total Potassium (\%) & $0.40 \pm 0.15$ \\
\hline Fe (ppm) & $240.5 \pm 32.2$ \\
\hline $\mathrm{Mn}(\mathrm{ppm})$ & $6.2 \pm 0.3$ \\
\hline $\mathrm{Cu}(\mathrm{ppm})$ & $3.5 \pm 0.5$ \\
\hline $\mathrm{Zn}(\mathrm{ppm})$ & $1.8 \pm 0.3$ \\
\hline
\end{tabular}

Value represents percentage of dry matter

Value represents mean $\pm S E(n=3)$

Table II. Chemical Properties of fish hydrolysate as bio fertilizer

\begin{tabular}{|l|l|}
\hline Parameter & Composition \\
\hline $\mathrm{p}^{\mathrm{H}}$ & $4.25 \pm 0.2$ \\
\hline $\mathrm{mv}$ & $162.0 \pm 11.0$ \\
\hline Organic Carbon $(\%)$ & $2.2 \pm 0.2$ \\
\hline Available nitrogen $(\mathrm{mg})$ in $100 \mathrm{~mL}$ & $392 \pm 0.21$ \\
\hline Available Phosphorus $(\mathrm{mg})$ in $100 \mathrm{~mL}$ & $10 \pm 0.5$ \\
\hline C/N ratio & 1.5 \\
\hline
\end{tabular}

\subsection{Preservation of Plankton Samples}

Plankton species were collected in a weekly basis from each experimental jars. The samples were fixed with $2 \mathrm{ml}$ of Lugols iodine (1:1000) for sedimentation and preservation of planktons. After 2-3 days the supernatant was carefully siphoned out and the volume was made upto $50 \mathrm{ml}$. the concentrated preserved plankton samples were analysed on a Sedgewick-Rafter counting cell (SRCell) under compound microscope with digital photography (APHA, 1989).

\subsection{Enumeration and Identification of Plankton}

For each sample, $1 \mathrm{ml}$ of sub-sample was transferred to the cell. From 10 randomly selected cell, square of the planktonic organisms were enumerated. The plankton was further identified upto genus level following the guidelines of (Bellinger, 1992; Needham and Needham 1972). The plankton abundance in the original volume was then computed using the formula by Stirling (1985).

$$
\mathrm{N}=\frac{A \times 100 \times C}{V \times F \times L}
$$

Where,

$\mathrm{N}=$ Number of plankton cells or units per litre of sample

$\mathrm{A}=$ Total number of plankton counted

$\mathrm{C}=$ Volume of final concentrate of the sample in $\mathrm{ml}$

$\mathrm{V}=$ Volume of a field in cubic $\mathrm{mm}$

$\mathrm{F}=$ Number of field counted

$\mathrm{L}=$ Volume of original water in Litre

\section{RESULTS AND DISCUSSION}

\subsection{Chlorophyll Production}

Table- 3 shows the growth of chlorophyll in the fish hydrolysate treated pond water. The maximum cell density was observed on $0.25 \mathrm{ml} \mathrm{L}^{-1}$ concentration. Highest chlorophyll a concentration was observed which showed a gradual increase as the time elapsed. Lower and higher concentrations of fish hydrolysate did not produce effective result in chlorophyll production. Fish hydrolysate $0.25 \mathrm{ml}$ $\mathrm{L}^{-1}$ dose increased the chlorophyll-a concentration ranged between 0.37-2.67 $\mu \mathrm{g} \mathrm{L}^{-1}$, a gradual increase was noted between 0-28 days. Similar results have been reported by Sipauba- Tavares et al., (2006). 
B.B. Sahu et al.

Table III. Generic status of plankton available in fish hydrolysate treated pond water

\begin{tabular}{|l|l|l|l|l|l|}
\hline Phytoplankton & Desmidiaceae & Bacillariophyceae & Zooplanktons & Crustacea & Rotrifera \\
\hline Chlorophyceae & Cyanophceae & Desta & Daphnia & Rotifers \\
\hline Ankistrodesmus & Anabaena & Closterium & Melosira & Sida & \\
\hline Botryoccoccus & Phormidium & Desmidium & Cocconies & Diaptomus & \\
\hline Chaetophora & Polycystis & Docidium & Diatoms & Cyclops & \\
\hline Cladophora & Rivularia & Gonatozygon & Navicula & Cypridopsis & \\
\hline Protococcus & Spirulina & Micrasterias & Synedra & Copepods & \\
\hline Scenedesmus & Merismopedia & Netrium & Amphora & & \\
\hline Spirogyra & Oscillatoria & Spirotaenia & Tabelaria & & \\
\hline Zygnema & & & Stephanodiscus & & \\
\hline Crucigenia & & & & & \\
\hline
\end{tabular}

Mean values of some water quality parameters are presented in Table 4. The presence of high amount of ammonia, carbon dioxide indicates a eutrophic nature, $\mathrm{pH}$ values in all the fish hydrolysate treatment ranged from 6.4 to 8.2. In heavily dosed fish hydrolysate jars, $\mathrm{pH}$ values increased up to 7.8 to 8.2 indicating water quality deterioration. In $0.25 \mathrm{ml} \mathrm{L}^{-1}$ fish hydrolysate treatment, $\mathrm{pH}$ was maintained at 7.5 to 7.7 indicating optimum value for vigorous plankton production.

Table IV. Percentile value of abundance of planktons during different periods of experiment

\begin{tabular}{|c|c|c|c|c|c|c|}
\hline $\begin{array}{l}\text { Sl. } \\
\text { No }\end{array}$ & $\begin{array}{l}\text { Names of the frequently available } \\
\text { plankton species in percentage }\end{array}$ & O days & 07 days & 14 days & 21 days & 28 days \\
\hline & \multicolumn{6}{|l|}{ Green algae } \\
\hline 1 & Ankistrodesmus & 33.3 & 46.6 & 60 & 73.3 & 66.6 \\
\hline 2 & Botryoccoccus & 53.3 & 33.3 & 66.6 & 86.6 & 80.0 \\
\hline 3 & Chaetophora & 20 & 26.6 & 40 & 46.6 & 46.6 \\
\hline 4 & Cladophora & 26.6 & 40 & 40 & 40 & 93.3 \\
\hline 5 & Protococcus & 33.3 & 33.3 & 80 & 100 & 53.3 \\
\hline 6 & Scenedesmus & 26.6 & 26.6 & 40 & 53.3 & 40.0 \\
\hline 7 & Spirogyra & 33.3 & 20 & 33.3 & 33.3 & 40.0 \\
\hline 8 & Zygnema & 33.3 & 53.3 & 46.6 & 53.3 & 53.3 \\
\hline \multirow[t]{2}{*}{9} & Crucigenia & 60 & 66.6 & 80 & 100 & 86.6 \\
\hline & \multicolumn{6}{|l|}{ Blue - Green Algae } \\
\hline 10 & Anabaena & 13.3 & 26.6 & 20 & 26.6 & 20.0 \\
\hline 11 & Phormidium & 20 & 33.3 & 13.3 & 33.3 & 26.6 \\
\hline 12 & Polycystis & 13.3 & 13.3 & 13.3 & 13.3 & 20.0 \\
\hline 13 & Rivularia & 26.6 & 13.3 & 40 & 60 & 60.0 \\
\hline 14 & Spirulina & 20 & 20 & 20 & 20 & 26.6 \\
\hline 15 & Merismopedia & 20 & 26.6 & 26.6 & 40 & 46.6 \\
\hline \multirow[t]{2}{*}{16} & Oscillatoria & 13.3 & 33.3 & 33.3 & 33.3 & 33.3 \\
\hline & \multicolumn{6}{|l|}{ Desmids (Desmidiaceae) } \\
\hline 17 & Closterium & 13.3 & 20 & 33.3 & 33.3 & 40.0 \\
\hline 18 & Desmidium & 20 & 20 & 26.6 & 40 & 33.3 \\
\hline 19 & Docidium & 20 & 20 & 20 & 20 & 20.0 \\
\hline 20 & Gonatozygon & 20 & 26.6 & 33.3 & 46.6 & 53.3 \\
\hline 21 & Micrasterias & 6.6 & 6.6 & 13.3 & 13.3 & 13.3 \\
\hline 22 & Netrium & 6.6 & 6.6 & 6.6 & 6.6 & 20.0 \\
\hline \multirow[t]{2}{*}{23} & Spirotaenia & 13.3 & 13.3 & 20 & 20 & 26.6 \\
\hline & \multicolumn{6}{|l|}{ Diatoms (Bacillariophyceae) } \\
\hline 24 & Melosira & 26.6 & 40 & 53.3 & 66.6 & 73.3 \\
\hline 25 & Cocconies & 13.3 & 13.3 & 13.3 & 13.3 & 20.0 \\
\hline 26 & Diatoms & 26.6 & 40 & 33.3 & 40 & 40.0 \\
\hline 27 & Navicula & 33.3 & 66.6 & 80 & 93.3 & 100.0 \\
\hline 28 & Synedra & 13.3 & 26.6 & 20 & 26.6 & 40.0 \\
\hline 29 & Amphora & 20 & 20 & 13.3 & 20 & 20.0 \\
\hline 30 & Tabelaria & 20 & 33.3 & 26.6 & 40 & 40.0 \\
\hline \multirow[t]{2}{*}{31} & Stephanodiscus & 20 & 20 & 13.3 & 20 & 20.0 \\
\hline & \multicolumn{6}{|l|}{ Zooplanktons } \\
\hline 32 & Daphnia & 20 & 26.6 & 20 & 33.3 & 46.6 \\
\hline 33 & Sida & 20 & 20 & 20 & 20 & 26.6 \\
\hline 34 & Diaptomus & 13.3 & 40 & 40 & 40 & 33.3 \\
\hline 35 & Cyclops & 33.3 & 46.6 & 66.6 & 93.3 & 100.0 \\
\hline 36 & Cypridopsis & 40 & 53.3 & 53.3 & 73.3 & 80.0 \\
\hline 37 & Copepods & 46.6 & 40 & 53.3 & 80 & 93.3 \\
\hline 38 & Rotifers & 26.6 & 26.6 & 20 & 26.6 & 46.6 \\
\hline
\end{tabular}




\subsection{Generic Status of Planktons Available in Fish Hydrolysate Treated Jars}

Generic plankton abundance in the experimental jars comprised of five groups (Table 5) consisting of 38 genera. The total planktonic organisms mainly composed of four groups of phytoplankton and one group of zooplankton. Some 31 genera of phytoplankton belonging to chlorophyce (9) cyanophyce (7) bacillariophyceae (8). Seven genera of zooplanktons were also identified.

Table V. Mean values of some Physico-chemical parameters in fish hydrolysate treated experimental jar water

\begin{tabular}{|c|c|c|c|c|c|c|c|}
\hline Parameters & Days & $\begin{array}{c}\text { T }-1 \\
\left(0.01 \mathrm{ml} \mathrm{L} \mathbf{L}^{-1}\right)\end{array}$ & $\begin{array}{c}\text { T-2 } \\
\left(0.05 \mathrm{ml} \mathrm{L}^{-1}\right)\end{array}$ & $\begin{array}{c}\mathrm{T}-3 \\
\left(0.25 \mathrm{ml} \mathrm{L}^{-1}\right)\end{array}$ & $\begin{array}{c}T-4 \\
\left(1.25 \mathrm{ml} \mathrm{L}^{-1}\right)\end{array}$ & $\begin{array}{c}\mathrm{T}-5 \\
\left(6.25 \mathrm{ml} \mathrm{L}{ }^{-1}\right)\end{array}$ & Control \\
\hline \multirow{5}{*}{$\begin{array}{l}\text { Temperature } \\
(\mathrm{oC})\end{array}$} & 0 & 25.1 & 25.3 & 25.2 & 25.1 & 25.1 & 25.3 \\
\hline & 7 & 26.3 & 26.1 & 25.8 & 27.0 & 27.3 & 26 \\
\hline & 14 & 26.3 & 26.4 & 26.0 & 26.5 & 26.7 & 26.2 \\
\hline & 21 & 27.1 & 27.2 & 27.03 & 26.9 & 26.4 & 26.5 \\
\hline & 28 & 26.3 & 26.0 & 26.4 & 26.8 & 26.4 & 26.9 \\
\hline \multirow[t]{5}{*}{$\mathrm{pH}$} & 0 & 6.4 & 6.4 & 6.4 & 6.4 & 6.4 & 6.4 \\
\hline & 7 & 7.4 & 7.5 & 7.7 & 7.8 & 8.2 & 6.4 \\
\hline & 14 & 7.5 & 7.4 & 7.6 & 7.4 & 7.8 & 6.5 \\
\hline & 21 & 7.4 & 7.3 & 7.5 & 7.1 & 6.7 & 7.5 \\
\hline & 28 & 7.1 & 6.9 & 6.7 & 7.0 & 6.9 & 6.8 \\
\hline \multirow{5}{*}{$\begin{array}{l}\text { Conductivity }(\mu s \\
/ \mathrm{cm})\end{array}$} & 0 & 304.0 & 311.6 & 312.0 & 318.3 & 307.7 & 304.0 \\
\hline & 7 & 368.3 & 335.3 & 328.3 & 286.3 & 311.0 & 319.0 \\
\hline & 14 & 289.7 & 293.0 & 342.3 & 190.0 & 130.3 & 323.0 \\
\hline & 21 & 323.3 & 321.7 & 346.3 & 156.3 & 141.7 & 331.0 \\
\hline & 28 & 308.7 & 351.0 & 355.3 & 349.0 & 263.3 & 346.0 \\
\hline \multirow{5}{*}{$\begin{array}{l}\text { Alkalinity } \\
(\mathrm{mg} / \mathrm{L})\end{array}$} & 0 & 60.7 & 61.3 & 61.3 & 61.3 & 63.3 & 62.0 \\
\hline & 7 & 62.0 & 61.3 & 62.7 & 58.0 & 56.0 & 64.0 \\
\hline & 14 & 63.3 & 75.3 & 80.0 & 56.0 & 56.7 & 64.0 \\
\hline & 21 & 64.7 & 79.3 & 82.7 & 55.3 & 51.3 & 66.0 \\
\hline & 28 & 64.0 & 82.7 & 86.0 & 54.7 & 73.3 & 64.0 \\
\hline \multirow{5}{*}{$\begin{array}{l}\text { Hardness } \\
(\mathrm{mg} / \mathrm{L})\end{array}$} & 0 & 40.7 & 40.7 & 40.0 & 41.3 & \begin{tabular}{|l|}
41.3 \\
\end{tabular} & 40.0 \\
\hline & 7 & 38.7 & 38.0 & 38.7 & 38.7 & 44.0 & 36.0 \\
\hline & 14 & 39.3 & 40.6 & 46.0 & 44.7 & 64.7 & 36.0 \\
\hline & 21 & 111.3 & 82.6 & 80.6 & 122.0 & 151.3 & 90.0 \\
\hline & 28 & 83.3 & 82.7 & 86.7 & 141.3 & 159.3 & 88.0 \\
\hline
\end{tabular}

\subsection{Relative Abundance of Phytoplankton and Zooplankton in FH Treated Jars}

The main phytoplankton groups recorded were Chlorophyce, Bacillariophyce (diatoms) cyanophyce and Desmidiace in the order of merit in $0.25 \mathrm{ml} \mathrm{L}^{-1}$ fish hydrolysate treated jars (Table 6, Figure 1). The percentage composition of each phytoplankton group was as follows (Table 7). In chlorophyce groups highest percentage of phytoplankton recorded were cladophora (93.3), crucigenia (86.6) and Botryococcus (80.6) respectively on 28 days of inoculation.

Table VI. Mean values of some Physico-chemical parameters in fish hydrolysate treated experimental jar water

\begin{tabular}{|c|c|c|c|c|c|c|c|}
\hline Parameters & Days & $\begin{array}{c}\mathrm{T}-\mathbf{1} \\
\left(0.01 \mathrm{ml} \mathrm{L}^{-1}\right)\end{array}$ & $\begin{array}{c}\mathrm{T}-2 \\
\left(0.05 \mathrm{ml} \mathrm{L}^{-1}\right)\end{array}$ & $\begin{array}{c}\mathrm{T}-3 \\
\left(0.25 \mathrm{ml} \mathrm{L}^{-1}\right)\end{array}$ & $\begin{array}{c}T-4 \\
\left(1.25 \mathrm{ml} \mathrm{L}^{-1}\right)\end{array}$ & $\begin{array}{c}\mathrm{T}-5 \\
\left.(6.25 \mathrm{ml} \mathrm{L})^{-1}\right)\end{array}$ & Control \\
\hline \multirow[t]{5}{*}{$\mathrm{CO}_{2}(\mathrm{mg} / \mathrm{L})$} & 0 & 2.0 & 2.0 & 2.0 & 2. & 2.0 & 2 \\
\hline & 7 & 4.7 & 5.3 & 5.3 & 7.3 & 6.7 & 4 \\
\hline & 14 & 4.0 & 4.7 & 5.3 & 8.7 & 6.7 & 4 \\
\hline & 21 & 6.0 & 4.7 & 6.7 & 10.0 & 10.0 & 4 \\
\hline & 28 & 4.0 & 4.0 & 6.0 & 8.7 & 7.3 & 4 \\
\hline \multirow{5}{*}{$\begin{array}{l}\text { Ammonia } \\
(\mathrm{mg} / \mathrm{L})\end{array}$} & 0 & 0.2 & 0.2 & 0.2 & 0.2 & 0.2 & 0.2 \\
\hline & 7 & 0.2 & 0.1 & 0.0 & 3.2 & 3.6 & 0.2 \\
\hline & 14 & 0.2 & 0.1 & 0.1 & 3.2 & 3.6 & 0.2 \\
\hline & 21 & 0.3 & 0.2 & 0.3 & 1.0 & 3.6 & 0.9 \\
\hline & 28 & 0.3 & 0.2 & 0.2 & 2.0 & 3.6 & 0.8 \\
\hline \multirow[t]{5}{*}{ Nitrate $(\mathrm{mg} / \mathrm{L})$} & 0 & 0.0 & 0.0 & 0.0 & 0.0 & 0.0 & 0.0 \\
\hline & 7 & 0.0 & 0 & 0.0 & 0.0 & 0.0 & 0.0 \\
\hline & 14 & 0.0 & 0 & 0 & 0.0 & 0.0 & 0.0 \\
\hline & 21 & 0.0 & 0 & 0 & 0.0 & 0.0 & 0.0 \\
\hline & 28 & 0.0 & 0 & 0 & 0.0 & 0.0 & 0.0 \\
\hline \multirow[t]{5}{*}{ Nitrite $(\mathrm{mg} / \mathrm{L})$} & 0 & 0.5 & 0.5 & 0.5 & 0.0 & 0.0 & 0.5 \\
\hline & 7 & 0.1 & 0.1 & 0.1 & 0.1 & 0.0 & 0.5 \\
\hline & 14 & 0.1 & 0.1 & 0.1 & 0.1 & 0.0 & 0.5 \\
\hline & 21 & 0.1 & 0.1 & 0.1 & 0.1 & 0.0 & 0.1 \\
\hline & 28 & 0.1 & 0.1 & 0.1 & 0.1 & 0.0 & 0.0 \\
\hline
\end{tabular}




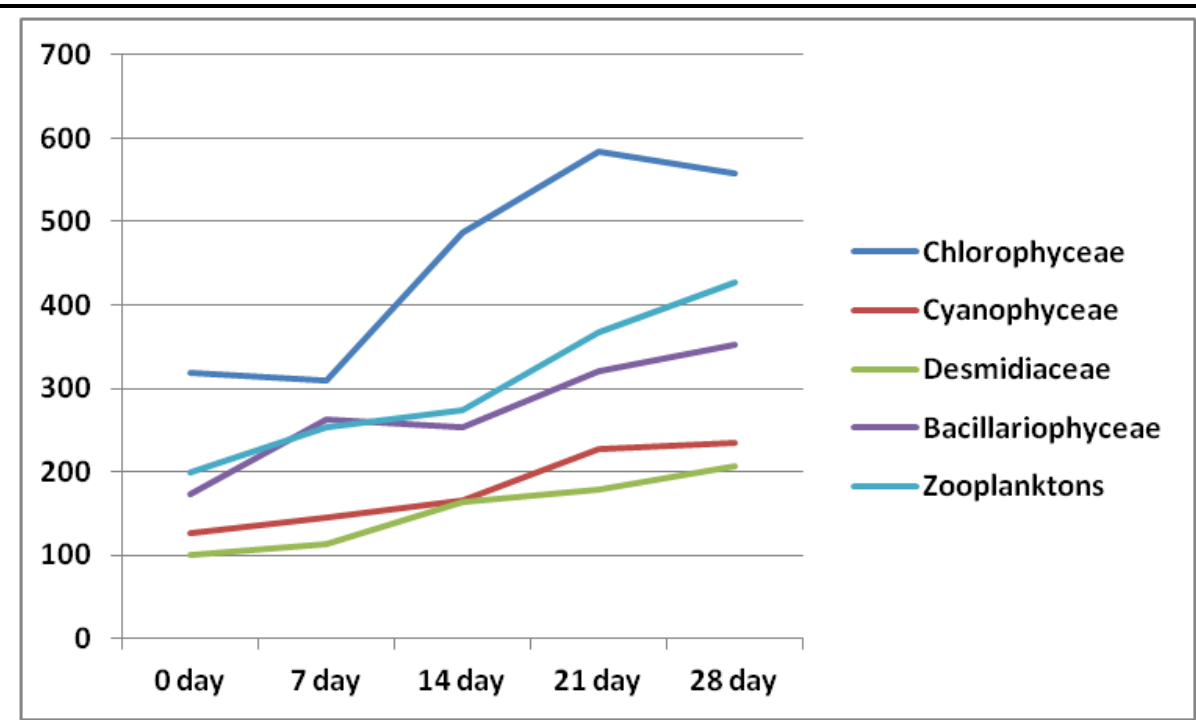

Fig I. Total abundance of generic phytoplankton and zooplankton species in hydrolysate treated jar water

Table VII. Mean values of chlorophyll $(\mu \mathrm{gm} / \mathrm{L})$ in various treatments

\begin{tabular}{|c|c|c|c|c|c|c|}
\hline Tank No. & Conc. $\left(\mathrm{ml} \mathrm{L}^{-1}\right)$ & Days & Chlorophyll a & Chlorophyll b & Chlorophyll c & Total Chlorophyll \\
\hline \multirow{5}{*}{ T - 1} & \multirow{5}{*}{0.01} & 0 & $0.37 \pm 0.01$ & $0.44 \pm 0.02$ & $0.93 \pm 0.02$ & $0.30 \pm 0.03$ \\
\hline & & 7 & $0.36 \pm 0.01$ & $0.59 \pm 0.02$ & $1.35 \pm 0.06$ & $0.40 \pm 0.03$ \\
\hline & & 14 & $0.73 \pm 0.03$ & $0.64 \pm 0.07$ & $1.77 \pm 0.08$ & $0.55 \pm 0.03$ \\
\hline & & 21 & $0.77 \pm 0.04$ & $0.35 \pm 0.03$ & $4.92 \pm 0.08$ & $1.04 \pm 0.07$ \\
\hline & & 28 & $0.86 \pm 0.02$ & $0.51 \pm 0.04$ & $4.24 \pm 0.12$ & $0.97 \pm 0.04$ \\
\hline \multirow[t]{5}{*}{$\mathbf{T}-\mathbf{2}$} & \multirow{5}{*}{0.05} & 0 & $0.37 \pm 0.01$ & $0.44 \pm 0.02$ & $0.93 \pm 0.02$ & $0.30 \pm 0.03$ \\
\hline & & 7 & $0.36 \pm 0.03$ & $0.56 \pm 0.03$ & $1.37 \pm 0.03$ & $0.40 \pm 0.03$ \\
\hline & & 14 & $0.97 \pm 0.03$ & $0.63 \pm 0.02$ & $1.78 \pm 0.02$ & $0.58 \pm 0.04$ \\
\hline & & 21 & $1.57 \pm 0.03$ & $1.45 \pm 0.02$ & $1.39 \pm 0.02$ & $0.76 \pm 0.05$ \\
\hline & & 28 & $1.85 \pm 0.03$ & $1.70 \pm 0.06$ & $1.48 \pm 0.02$ & $0.87 \pm 0.05$ \\
\hline \multirow[t]{5}{*}{ T - 3} & \multirow{5}{*}{0.25} & 0 & $0.37 \pm 0.01$ & $0.44 \pm 0.02$ & $0.93 \pm 0.02$ & $0.3 \pm 0.03$ \\
\hline & & 7 & $0.38 \pm 0.02$ & $0.57 \pm 0.04$ & $1.39 \pm 0.02$ & $0.41 \pm 0.02$ \\
\hline & & 14 & $1.86 \pm 0.04$ & $0.87 \pm 0.03$ & $1.80 \pm 0.02$ & $0.79 \pm 0.05$ \\
\hline & & 21 & $2.64 \pm 0.04$ & $1.47 \pm 0.01$ & $2.51 \pm 0.39$ & $0.79 \pm 0.05$ \\
\hline & & 28 & $2.67 \pm 0.03$ & $1.65 \pm 0.04$ & $2.08 \pm 0.02$ & $1.14 \pm 0.04$ \\
\hline \multirow[t]{5}{*}{$T-4$} & \multirow{5}{*}{1.25} & 0 & $0.37 \pm 0.01$ & $0.44 \pm 0.02$ & $0.93 \pm 0.02$ & $0.3 \pm 0.02$ \\
\hline & & 7 & $0.41 \pm 0.02$ & $0.54 \pm 0.03$ & $1.36 \pm 0.01$ & $0.52 \pm 0.02$ \\
\hline & & 14 & $1.04 \pm 0.05$ & $0.77 \pm 0.01$ & $1.17 \pm 0.03$ & $0.52 \pm 0.03$ \\
\hline & & 21 & $1.85 \pm 0.03$ & $1.97 \pm 0.02$ & $2.08 \pm 0.05$ & $1.02 \pm 0.08$ \\
\hline & & 28 & $0.63 \pm 0.01$ & $0.82 \pm 0.02$ & $1.62 \pm 0.02$ & $0.53 \pm 0.03$ \\
\hline \multirow[t]{5}{*}{$\mathbf{T}-\mathbf{5}$} & \multirow{5}{*}{6.25} & 0 & $0.37 \pm 0.01$ & $0.44 \pm 0.02$ & $0.93 \pm 0.02$ & $0.3 \pm 0.03$ \\
\hline & & 7 & $0.52 \pm 0.05$ & $0.63 \pm 0.02$ & $1.60 \pm 0.06$ & $0.48 \pm 0.05$ \\
\hline & & 14 & $0.67 \pm 0.01$ & $0.62 \pm 0.02$ & $1.11 \pm 0.02$ & $0.41 \pm 0.02$ \\
\hline & & 21 & $0.84 \pm 0.03$ & $0.54 \pm 0.05$ & $1.53 \pm 0.02$ & $0.5 \pm 0.04$ \\
\hline & & 28 & $0.66 \pm 0.03$ & $0.54 \pm 0.03$ & $0.94 \pm 0.04$ & $0.37 \pm 0.02$ \\
\hline \multirow[t]{5}{*}{ Control } & \multirow{5}{*}{ NIL } & 0 & $0.37 \pm 0.01$ & $0.44 \pm 0.02$ & $0.93 \pm 0.02$ & $1.11 \pm 0.06$ \\
\hline & & 7 & $1.14 \pm 0.06$ & $0.47 \pm 0.04$ & $2.37 \pm 0.05$ & $0.30 \pm 0.03$ \\
\hline & & 14 & $0.42 \pm 0.03$ & $0.53 \pm 0.02$ & $1.36 \pm 0.08$ & $0.69 \pm 0.04$ \\
\hline & & 21 & $0.46 \pm 0.02$ & $0.50 \pm 0.03$ & $4.06 \pm 0.05$ & $0.87 \pm 0.06$ \\
\hline & & 28 & $0.54 \pm 0.07$ & $0.68 \pm 0.03$ & $3.56 \pm 0.41$ & $0.83 \pm 0.10$ \\
\hline
\end{tabular}

Table VIII. Total abundance of generic phytoplankton and zooplankton species in hydrolysate treated jar water

\begin{tabular}{|l|l|l|l|l|l|}
\hline $\begin{array}{l}\text { Sl. No. of plankton } \\
\text { Species }\end{array}$ & $\mathbf{0}$ day & $\mathbf{7}$ day & $\mathbf{1 4}$ day & $\mathbf{2 1}$ day & $\mathbf{2 8 ~ d a y ~}$ \\
\hline Chlorophyceae & $319.7 \times 10^{3}$ & $310.1 \times 10^{3}$ & $486.5 \times 10^{3}$ & $583.4 \times 10^{3}$ & $557.7 \times 10^{3}$ \\
\hline Cyanophyceae & $126.5 \times 10^{3}$ & $144.1 \times 10^{3}$ & $165.5 \times 10^{3}$ & $226.5 \times 10^{3}$ & $233.8 \times 10^{3}$ \\
\hline Desmidiaceae & $99.8 \times 10^{3}$ & $113.1 \times 10^{3}$ & $163.1 \times 10^{3}$ & $177.8 \times 10^{3}$ & $206.5 \times 10^{3}$ \\
\hline Bacillariophyceae & $173.1 \times 10^{3}$ & $261.8 \times 10^{3}$ & $253.1 \times 10^{3}$ & $319.8 \times 10^{3}$ & $352.3 \times 10^{3}$ \\
\hline Zooplanktons & $199.8 \times 10^{3}$ & $253.1 \times 10^{3}$ & $273.2 \times 10^{3}$ & $366.5 \times 10^{3}$ & $426.4 \times 10^{3}$ \\
\hline
\end{tabular}


In Bacillariophyceae, Navicula (100\%) Melosira (73.3\%) Diatoms and Synedra (40\%). In Desmidaceae group Gonatozygon (53.3\%) and Closterium (40\%) was recorded. Cyanophyce groups Rivularia (60\%) and Merismopedia (46.6\%) were recorded. Zooplankton groups had Cyclops (100\%), Copepods (93.3\%) and Cypridopsis (80\%) were recorded.

Optimal fertilization rate is the amount of organic matter that may be cost effective, eco-friendly and utilized in a pond ecosystem without any harmful effect on water quality and fish growth.

Phytoplankton requires both macronutrients and micronutrients for their growth. Fish hydrolysate is an organic fertilizer consisting of macronutrients, micronutrients and secondary elements in a natural proportion in liquid biofertilizer. The observed highest number of phytoplankton and zooplankton in T-3 showed that micronutrients and macronutrients at a proper dose are very much essential. (Boyd, 1979).

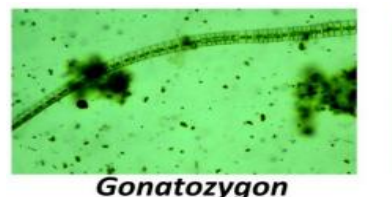

Gonatozygon

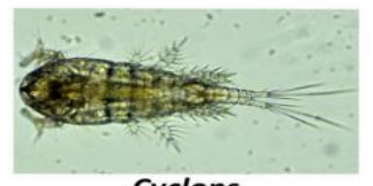

Cyclops

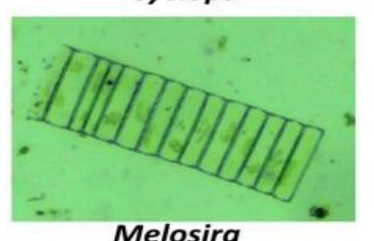

Melosira

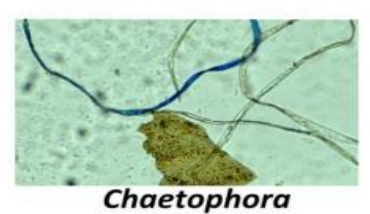

Chaetophora
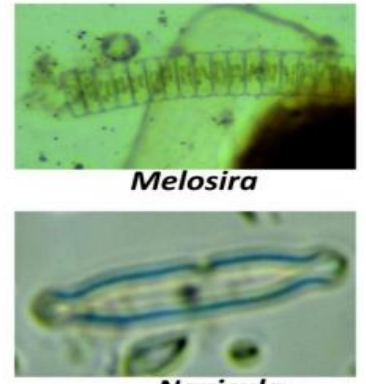

Navicula
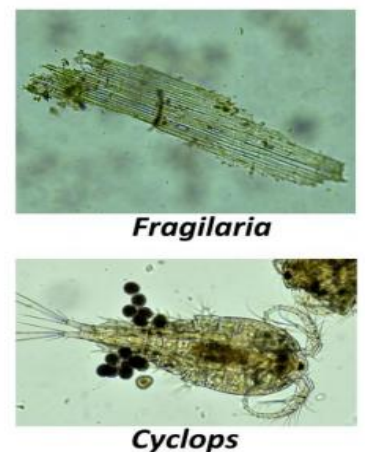

Fig IIa. Quality zooplankton \& phytoplankton production in $0.25 \mathrm{ml} \mathrm{L}{ }^{-1} \mathrm{FH}$ treated T-3 glass jars

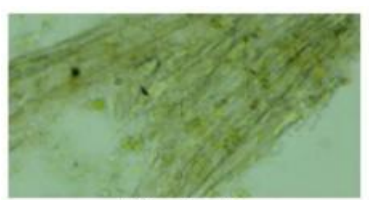

Rivularia

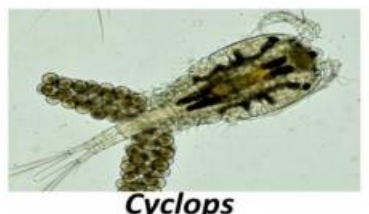

Cyclops

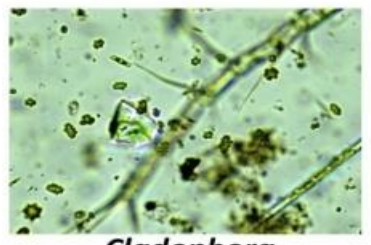

Cladophora

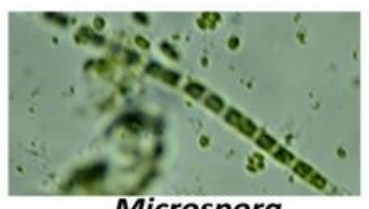

Microspora

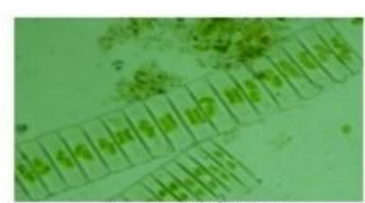

Desmidium

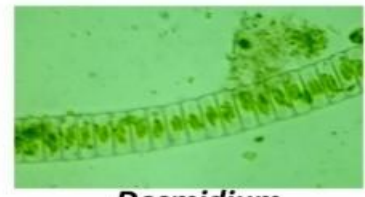

Desmidium

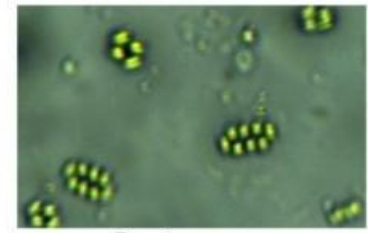

Protococcus

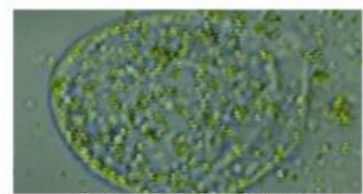

Coelosphaerium

Fig IIb. Quality zooplankton \& phytoplankton production in $0.25 \mathrm{ml} \mathrm{L} \mathrm{L}^{-1} \mathrm{FH}$ treated T-3 glass jars 


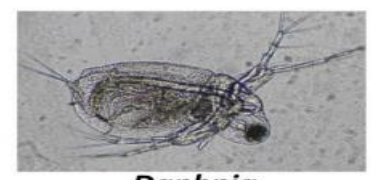

Daphnia
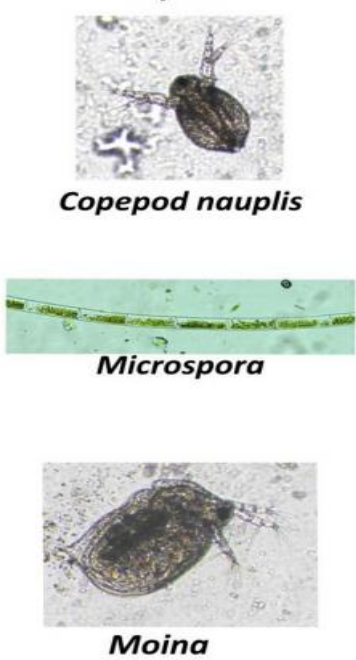

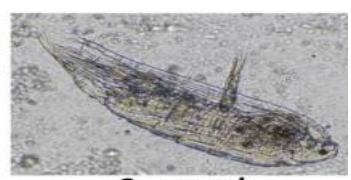

Copepods
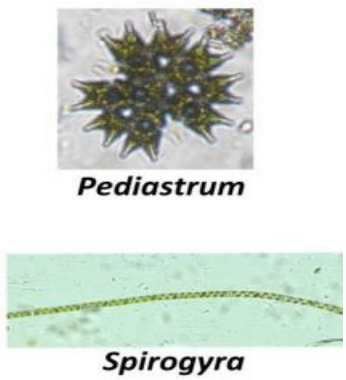

Spirogyra

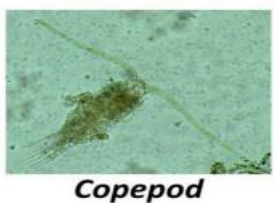

Fig IIc. Quality zooplankton \& phytoplankton production in $0.25 \mathrm{ml} \mathrm{L} \mathrm{L}^{-1} \mathrm{FH}$ treated T-3 glass jars

\subsection{Physicochemical Parameters of Water Quality}

The water quality parameters such as temperature, $\mathrm{pH}$, alkalinity, $\mathrm{CO}_{2}$, ammonia, nitrate and nitrite remained congenial for fish culture point of view in $0.25 \mathrm{ml} \mathrm{L}^{-1}$ fish hydrolysate treated jars. Boyd (1979) reported similar results in freshwater aquaculture ponds. The physicochemical condition was within the suitable range and biological production was higher in T-3, where $0.25 \mathrm{ml} \mathrm{L}^{-1}$ fish hydrolysate was used as organic fertilizer. Therefore, it may be recommended for higher productivity of a water body. Total phytoplankton population growth in hydrolysate treated water than the control pond water due to micronutrients nitrogen, phosphorus and potassium and trace elements present in the fish hydrolysate. A significant amount of iron $(\mathrm{Fe})$ present in the fish hydrolysate. Iron is an especially essential element for the growth of phytoplankton and its deficiency is well known to suppress primary productivity in both freshwater and marine ecosystem (Naito et al., 2006). Iron has the potential to control the growth and composition of phytoplankton communities. (Noiri et al., 2005)

\subsection{Generic Status of Planktons Available in Fish Hydrolysate Treated Jars}

Production of an unpredictable mixture of algal is greatly covered by application of fish hydrolysate $0.25 \mathrm{ml} \mathrm{L}^{-1}$ (T-3) throughout the experimental pond. Phytoplanton require both macronutrients and micronutrients for their growth. Fish hydrolysate contains both macro and micronutrients in a balanced form. It contains iron, manganese, copper and zinc as micronutrients N:P:K::1.5:0.5:0.4 as macronutrients. (Davies et al., 2006)

Generic plankton abundance in the experiment with T-3 doses comprised of five groups, 4 groups of phytoplankton and 1 group of zooplankton, consisting of 31 genera. Some 31 genera of phytoplankton belonging to chlorophyceae (9) Cyanophyceae (7) Bacillariophyce (8). Seven genera of zooplankton were also identified. The Chlorophyceae group was the most representative, followed by bacillariophyceae and cyanophyceae. The zooplankton community consisted of cladocerans, copepods and rotifers and they are common or constant throughout the experiment. Planktonic organisms such as green algal (chlorophyta) copepod-calanoida were more abundant in organic fertilized fish ponds. Rotifers and chaoborus species densities were increased when inorganic fertilizers were used in the aquaculture ponds (Sipauba-Tavares et al., 2006)

\subsection{Relative Abundance of Phytoplankton and Zooplankton in FH Treated Jars}

In $0.25 \mathrm{ml} \mathrm{L}^{-1} \mathrm{FH}$ treated jars phytoplankton groups chlorophyceae; bacillariophyceae (diatoms) cyanophyceae and Desmidaceae were recorded in order of merit. Numerical density of phytoplankton implies that green algae occupied the first predominant pair and followed by the diatoms. Higher concentration of zooplankton organisms like rotifers and the positive indicators from fish rearing point of view. (Ayyappan et al., 1990; Mageed and Konsowa, 2002) 
Rotifers have shortest life span (12 days) and can reach their peak reproduction level in about 3-5 days. Cladocerans and copepod have similar lifespan of approximately 50 days. But Cladocerans require 14-15 days to reach peak reproduction capacity. However, copepods require 24 days. (Allan, 1976)

Application of FH at $0.25 \mathrm{ml} \mathrm{L}^{-1}$ dose had produced Cyclops (100\%) copepods (93.3\%) cypridopsis $(80 \%)$ in the experimental period. Similar results have been reported in zooplankton production by using biofertilizer enriched vermin-compost on production and growth of primary product.

\section{CONCLUSION}

From the present study it is concluded that water quality parameters were found within the optimum limit for primary production and freshwater aquaculture. The highest chlorophyll and plankton composition (phytoplankton and zooplanktons) was observed in T-3 $\left(0.25 \mathrm{ml} \mathrm{L}^{-1}\right)$ fish hydrolysate treatment and lowest value was recorded in control. We strongly recommended fish hydrolysate at the above concentration FH biofertilizer as successful organic manure an eco-friendly product for aquaculture practices. The dose has been calculated to be $20 \mathrm{~L} \mathrm{Ac}^{-1} \mathrm{~m}$ water body in 4 split doses with 15 days interval for aquaculture practices.

\section{ACKNOWLEDGEMENT}

The authors are thankful to the Director, ICAR-CIFA, Kausalyaganga, Bhubaneswar for providing the facilities for conducting the research and to Sidhartha mahali for photography and documentation assiatance.

\section{REFERENCES}

[1] Afonso MD and Bórquez R. 2002. Review of the treatment of seafood processing wastewaters and recovery of proteins therein by membrane separation processes: prospects of the ultrafiltration of wastewaters from the fish meal industry. Desalination 142, 29-45.

[2] Allan, J.D. (1976) Life history patterns in zooplankton. Am. Nat., 110, pp. 165-180.

[3] APHA 1989. Standard methods for examination of water and waste water, 12th edn.American Public Health Association, Washington D.C., $1452 \mathrm{pp}$

[4] Ayyappan, S., Pandey, B. K. and Tripathi, S. D. (1990) Nutritive quality of microbial communities as feed components in experimental carp culture. Indian J. Exp. Biol., 28(10), pp. 977--980.

[5] Begum, H., Najejo, N.T., Achakzai, G.D. and Ghotto, M. A. (2012) Impact of indiscriminate use of cow dung on the physico-chemical parameters of nursery ponds of the Nursery Ponds Of Chilya Fish Hatchery District Thatta, Sindh. Sindh Univ. Res. Jour. (Sci. Ser.), 44(3), pp. 355-360 .

[6] Bellinger, E.G. (1992) A key to common algae, The Institute of water and Environmental Management, London, pp. 138.

[7] Boyd CE (1979). Water quality in warm water fish ponds, Auburn University Agricultural, Experiment Station, Alabama, U.S.A, pp. 59.

[8] Chowdhury, MMR., Mondol, MRK. And Sarkar, C. (2007) Seasonal variation of plankton population of Borobilabeel in Rangpur district. University Journal of Zoology, Rajshahi University, 26(5), pp. 49--54.

[9] Davies, O. A., Alfred-Ockiya, J. F. and Asele, A. (2006). Induced growth of phytoplankton using two fertilizers (NPK and agrolyser) under laboratory conditions. African Journal of Biotechnology Vol. 5 (4), pp. 373-377

[10] Day AD and Katterman FRH. 1992. Sewage sludge provides plant growth factors in arid environments. J Arid Environ 23, 229-233.

[11] Gao M.T., Hirata M, Toorisaka E and Hano T. 2006. Acid-hydrolysis of fish wastes for lactic acid fermentation. Bioresour Technol 97, 2414-2420.

[12] Geiger, J.G. and C.J. Turners (1990) Pond fertilization and zooplankton management techniques for production of fingerling striped bass and hybrid striped bass in culture and propagation striped bass and its hybrid. 
[13] Hecky, R. E. and P. Kilham. (1988) Nutrient limitation of phytoplankton in freshwater and marine environments: A review of recent evidence on the effects of enrichment. Limnol. Oceanogr., 33, pp. 796--822.

[14] Jhingran, V.G. (1983) Fish and Fisheries of India. Hindustan Publication Corporation India, New Delhi, pp. 666.

[15] Kim JK, Dao VT, Kong IS and Lee HH.( 2010). Identification and characterization of microorganisms from earthworm viscera for the conversion of fish wastes into liquid fertilizer. Bioresour Technol 101, 5131-5136.

[16] Kumar, NJI. And Omen, C. (2009) Influence of limiting factors on phytoplankton and coliform population in an inundated, isolated wetland. Journal of Wetlands Ecology, 3, pp. 43--55.

[17] Mageed, A. A. and A. H. Konsowa. (2002) Relationship between phytoplankton, zooplankton and fish culture in a freshwater fish farm. Egyptian J. Aquatic. Biol. \& Fish., 6(2), pp. 183--206.

[18] Malik, S. (1992) Microbial population and zooplankton production potentialities of selected organic and chemical fertilizers. M. Sc. (Ag.) Thesis, Department of Limnology and Fisheries, RCA, Rajasthan Agriculture University, Udaipur, pp. 131.

[19] Martin Antonio M. and Paul L. Bemister (1994) Use of Peat Extract in the Ensiling of Fisheries Wastes Waste Management Research. 12 (November) 6, pp. 467--479.

[20] Mirza, M.J.A., Kadir, M.A., Nandi, C.S., and Nuruzzaman,N., (1990) Effects of cow dung on the physico chemical characteristics of the nursery ponds. Bangladesh J. Train. Devt. , 3(1), pp.83-89.

[21] Morris, J.E. and Mischeke, C.C. (1999) Plankton management for fish production ponds. Technical bulletin series 114, Ames, lowa.

[22] Moutin, T., Thingstad, TT.,Wambeke, FV., Marie, D., Slawyk, G., Raimbault, P. and Claustre, H. (2002) Does competition for nanomolecular phosphate supply explain the predominance of the cyanobacterium Synechococcus. Limnology and Oceanography, 47(5), pp. 1562-1567. http://dx.doi. org/10.4319/lo.2002.47.5.1562.

[23] Naito K., Suzuki M., Mito S., Hasegawa H., Matsui M. \& Imai I. (2006) Effects of the substances secreted from Closterium aciculare (Charophyceae, Chlorophyta) on the growth of freshwater phytoplankton under iron-deficient conditions. Plankton Benthos Res, 1(4), pp. 191-199.

[24] Needham, J. G. and Needham, R. P (1972) A guide to the study of freshwater biology. Holdenday, INC Cali 94 (III), U.S.A.

[25] Noiri Y, Kudo I, Kiyosawa H, Nishioka J, Tsuda A (2005) Influence of iron and temperature on growth, nutrient utilization ratios and phytoplankton species composition in the western subarctic Pacific Ocean during the SEEDS experiment. Prog. Oceanogr, 64, pp. 149--166.

[26] Olah, J., V.R.P. Sinha, S. Ayyappan, C.S. Purushothaman and S. Radheyshyam (1986) Primary production and fish yields in fish ponds under different management practices. Aquaculture, 58, pp. 111--122.

[27] Pennak, R. E. (1989) Freshwater invertebrates of United States: Protozoa to Mollusca (3rd eds.). New York: Wiley.

[28] Sahu, B. B., Barik, N. K., Mohapatra, B. C., Sahu, B. N., Sahu, H., Sahoo, P., Majhi, D., Biswal, N. C., Mohanty, P.K., Jayasankar,P. (2014) Valorization of fish processing waste through natural fermentation with molasses for preparation of bio fertilizer and bio supplement. JECET, 3(3), pp. 1849--1855.

[29] Senthil Kumar, D., Satheesh Kumar, P., Uthaya kumar, V. and G. Anbuganapathi (2014) Efficacy of Biofertilizer Enriched Flower Waste Vermicompost on Production and Growth of Primary Producers and Freshwater Aquarium Fishes. Global Veterinaria, 13(2), pp. 215--220.

[30] Sipaúba-Tavares, L.H., Baccarin., A.E. and Braga, F.M. de S. (2006) Limnological parameters and plankton community responds in Nile tilapia ponds under chicken dung and NPK (4-14-8). Fertilizers.Acta.Limnol.Bras. 18(3), pp. 335-346.

[31] Sipaúba-Tavares, LH.,Donadon, ARV. and Milan, RN. (2011) Water quality and plankton populations in an earthen polyculture pond. Braz. J. Biol.,71 (4) pp. 845-855. 
[32] Stirling, H.P. (1985) Chemical and biological methods of water analysis for aquaculturists, Institute of Aquaculture, University of Stirling, Scotland, pp. 119.

[33] Yamamato, K. (1960). Plankton rotation Japanese Inland. Hyd. Vol.XVI4, pp. 364--411.

\section{AUTHORS' BIOGRAPHY}

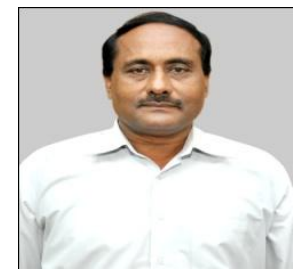

Dr. Binod Bihari Sahu, ICAR-Central Institute of Freshwater Aquaculture, Kausalyaganga, Bhubaneswar-751002, Odisha, India.

Dr. Bikash Chandra Mohapatra, ICAR-Central Institute of Freshwater Aquaculture, Kausalyaganga, Bhubaneswar, Odisha, India.

Mr. Nagesh Kumar Barik, ICAR-Central Institute of Freshwater Aquaculture, Kausalyaganga, Bhubaneswar, Odisha, India.

Mr. Hrushikesha Sahu, ICAR-Central Institute of Freshwater Aquaculture, Kausalyaganga, Bhubaneswar, Odisha, India.

Mr. Prithviraj Sahoo, ICAR-Central Institute of Freshwater Aquaculture, Kausalyaganga, Bhubaneswar, Odisha, India.

Mr. Nirmal Chandra Biswal, Dept. of Zoology, Utkal University, VaniVihar, Bhubaneswar, Odisha, India.

Dr. Prafulla Kumar Mohanty, HoD, Dept. of Zoology, Utkal University, Vani Vihar, Bhubaneswar, Odisha, India.

Dr. Utkal Laxmi Mohanty, ICAR-Central Institute of Freshwater Aquaculture, Kausalyaganga, Bhubaneswar, Odisha, India.

Mr. Sudeep Kumar Mahanta, ICAR-Central Institute of Freshwater Aquaculture, Kausalyaganga, Bhubaneswar, Odisha, India.

Dr. P. Jayasankar, Director, ICAR-Central Institute of Freshwater Aquaculture, Kausalyaganga, Bhubaneswar, Odisha, India. 